\title{
A REMARK ON REGULAR BANACH ALGEBRAS
}

\section{JOHN RAINWATER}

Let $A$ be a commutative semisimple regular Banach algebra with identity 1 and maximal ideal space $\mathfrak{T}$. For simplicity, we identify $A$ and $A^{\wedge}$, the Gelfand representatives of $A$ in $C(\mathscr{T T})$. Thus, $A$ is an algebra of continuous functions containing the constants on the compact Hausdorff space $\mathfrak{T}$. A well known consequence of the regularity of $A$ is the fact that any element of $C(\mathscr{T T})$ which is locally in $A$ is actually in $A$. (A function $f$ on $\mathfrak{T}$ is said to be locally in $A$ if for each $x$ in $\mathscr{T}$ there exists a neighborhood $U$ of $x$ and element $a$ in $A$ such that $f=a$ on $U$.) The purpose of this note is to prove a related result for certain subalgebras $B$ of $A$. We say that a subalgebra $B$ of $C(\mathfrak{T C})$ separates the points of $\mathscr{N}$ if to each $x, y$ in $\Re, x \neq y$, there exists $b$ in $B$ such that $b(x)=0$ and $b(y)=1$. (If $1 \in B$, this is the same as the usual definition of "separating".)

THEOREM. Let $B$ be a subalgebra (not necessarily closed) of the regular algebra $A$, which separates the points of $\mathfrak{T}$, and suppose that every element of $A$ is locally in $B$. Then $B=A$.

[Applied, in particular, to $A=C(X), X$ compact Hausdorff, this says that any separating subalgebra $B$ of $C(X)$ which yields the same "germs" as $C(X)$ at each $x$ in $X$ is necessarily all of $C(X)$.]

Proof. For any $a$ in $A$ and $x$ in $\mathscr{T}$ there is an element $b$ of $B$ with $b=a$ on some neighborhood $U$ of $x$. Thus, by compactness, there exist elements $b_{1}, \cdots, b_{n}$ of $B$ and an open covering $U_{1}, \cdots, U_{n}$ of $\mathfrak{T}$ for which $a=b_{i}$ on $U_{i}$. It will suffice to prove that subordinate to such a covering there exists a "partition of unity" $\left\{e_{1}, \cdots, e_{n}\right\}$ in $B$ (i.e., each $e_{i}$ vanishes off $U_{i}$, and $\sum e_{i}=1$ ). Indeed, we then observe that $a=\sum e_{i} b_{i}$ is in $B$.

Suppose that $x$ and $y$ are distinct points of $\mathfrak{T}$. By hypothesis, there exists $b$ in $B$ with $b(x)=0$ and $b(y)=1$, so $0 \notin b(W)$ for some compact neighborhood $W$ of $y$. Let $k W$ denote the ideal $\{a: a \in A, a(W)=0\}$. Since $A$ is regular, $W$ is the maximal ideal space of the quotient algebra $A / k W$ and the corresponding Gelfand representation is defined by $a+\left.k W \rightarrow a\right|_{w}$. Thus, $b$ gives rise to an invertible element of $A / k W$, so $a b=1$ on $W$ for some $a$ in $A$. But the element $a$ coincides with some $b^{\prime}$ in $B$ on a neighborhood of $y$, so $b b^{\prime}=1$ near $y$ while $b b^{\prime}(x)=0$. Now the element $1-b b^{\prime}$ is in $A$, vanishes in a neighborhood of $y$ and is 1

Received by the editors December 2, 1964. 
at $x$, so the same argument, applied to $1-b b^{\prime}$ and $x, y$ interchanged, shows that there exists $b^{\prime \prime}$ in $B$ such that $b^{\prime \prime}\left(1-b b^{\prime}\right)=1$ in a neighborhood of $x$. Thus, $e=b^{\prime \prime}-b^{\prime \prime} b b^{\prime}$ is in $B$, vanishes near $y$ (since $1-b b^{\prime}$ does) and is 1 near $x$. By a well-known argument [2] the existence of such elements $e$ of $B$ shows that $B$ is a normal algebra of functions on $\mathfrak{T}$ (in the obvious sense) so that the desired partitions of unity can be obtained as in [2].

Note that we need to assume that the points of $\mathscr{T}$ at which the elements of $A$ belong locally to $B$ comprise all of $\mathfrak{T}$ : Consider the subalgebra $B$ of $C([0,1])$ consisting of those functions which coincide with a polynomial near 0 . Also, $B$ must be an algebra and not just a subspace, as is shown by the subspace $B$ of $C\left(\left[-1,-\frac{1}{2}\right] \cup\left[\frac{1}{2}, 1\right]\right)$ consisting of all functions of the form $f+c$ where $c$ is a constant and $f$ as an odd function.

As is well known [1], if $A$ is a sup norm algebra, elements of $C(\mathfrak{T})$ belonging locally to $A$ need not belong to $A$; whether the analogue of our result is valid for sup norm algebras $A$ remains an open question.

\section{BIBLIOGRAPHY}

1. Eva Kallin, A nonlocal function algebra, Proc. Nat. Acad. Sci U.S.A. 49 (1963), 821-824 1953.

2. L. H. Loomis, Abstract harmonic analysis, Van Nostrand, Princeton, N. J.,

UNIVERSITY OF WASHINGTON 